\title{
What's wrong with "the mindful brain"? Moving past a neurocentric view of meditation
}

\author{
Michael Lifshitz \\ Department of Anthropology, Stanford University \\ Integrated Program in Neuroscience, McGill University \\ Evan Thompson \\ Department of Philosophy, University of British Columbia
}

In Amir Raz and Robert Thibault, eds., Casting Light on the Dark Side of Brain Imaging

(Academic Press, 2019)

\begin{abstract}
Meditation is trending right now. From classrooms and hospitals to business meetings and phone apps, our culture is enthralled by meditation as a powerful tool to train our brains and shape our private mental lives - to make us happier, more productive, and more peaceful on the inside. But meditation is not just about training our brains. It's a deeply social - and fundamentally embodied - collection of cultural practices. If we reduce meditative practices to just a set of brain patterns, we miss the richness of how these practices work and ignore much of what they have to teach us about our own subjective experience.
\end{abstract}

\section{Keywords}

Mindfulness; Meditation; Neuroscience; Enactive cognition; Embodied cognition

\section{MAIN TEXT}

Riding a growing wave of scientific data, mindfulness meditation has found its way to the forefront of popular culture. Mindfulness practices appeal to a wide spectrum of society, from stressed out office workers and college students to stock traders, doctors, government officials, and even infantry soldiers. These practices have come a long way from their humble beginnings at the outskirts of ancient Indian society. Mindfulness has emerged as a major force in global culture-enshrined in national medical guidelines, showcased on trendwatcher lists, and raking in over a billion dollars in profit each year ${ }^{1}$.

The growing mindfulness hype revolves around a particular view of mindfulness meditation as a kind of brain training ${ }^{2}$. At the heart of this trend lies a simple idea with apparently massive appeal: practicing meditation, we are told, literally rewires your brain ${ }^{3}$. It's a catchy idea: train 
your mind, change your brain ${ }^{4}$. But this idea has its problems, both empirical (as to the strength of the available evidence) and conceptual (as to whether it even makes sense to think of meditation in these terms).

In this chapter, we argue that the neurocentric view of mindfulness meditation, a perspective we call "the mindful brain", is a simplistic take on what meditation is and of how it works. Contrary to the neurocentic view, meditation is a deeply social, and fundamentally embodied collection of cultural practices. If we reduce meditative practices to a set of brain patterns, we miss the richness of how these practices work and ignore much of what they have to teach us about human experience 5 .

\section{The appeal of the mindful brain}

In today's language, mindfulness is understood as a state, or trainable skill, of paying attention in a particular way. To be mindful is to become aware of the ongoing stream of present-moment experience with an attitude of curiosity and acceptance. According to this popular understanding, mindfulness is about noticing what's going on with your thoughts, your body, and your emotions - taking stock of the subtle nuances of lived experience that we tend to gloss over in our busy lives. By training particular networks in our brains, we can learn to pay attention in this mindful way. It's not hard to see why this idea appeals to our contemporary sensibilities. We live in a wired world that relentlessly assaults us with complexity. Mindfulness seems to offer private access to a simpler mode of consciousness. It can give us a sense of control over our inner lives. The popular, yet misguided, idea that mindfulness resides in the brain suggests that the key to our happiness, peace, and productivity lies within. By controlling our brain, we take control of our own subjective well-being.

If there's one thing our contemporary culture values more than individual self-determination, it's tangible results. We put our faith in what we can measure. The concept of the mindful brain suggests that practicing mindfulness meditation really does something-something physical, concrete. The proof, apparently, is in the brain pudding. Studies suggest that Buddhist monks (the "Olympic athletes" of meditation) have thicker brains in all the right places, and that even busy westerners can thicken their brains after just a few weeks of daily meditation ${ }^{6}$. The implication is clear: if we are willing to meditate just a few minutes per day, we too can rewire our brains to gain more awareness and control over our own minds - to become happier, more productive, and more peaceful on the inside.

It's appealing to think that we might identify a specific brain signature of the mindful state. Then we might optimize meditation training to achieve that state more quickly and easily. We could skip all the fluff that has tended to come along with meditation (the religious cosmology, the moral doctrines, the bells and robes) and focus on what some people take to be the real essence: 
strengthening the brain's attention networks to achieve non-judgmental awareness of our own experience. Ostensibly, this optimized neuro-mindfulness approach will get us the results we want, and fast. Since the advent of the mindful brain, finding inner peace has never been easier. These days, you don't even need to seek out a meditation teacher or community-you can just download a mindfulness app or strap on a brain-sensing headband to ramp up your brainwaves. For a low cost, and just a few minutes of your time, you too can have your own mindful brain.

\section{Limitations of the mindful brain}

The finding that meditation changes your brain is often taken as a kind of proof that meditation really works. The tacit understanding seems to be that documenting the effects of meditation on the physical tissue of the brain makes these effects more substantial and reliable, more trustworthy-more real. But all mental activity is presumably reflected at the level of brain function, so it's hardly surprising that a change in mental behavior corresponds to a change in the brain. Any repetitive activity you do is likely to leave lasting traces on your brain. Learning to play an instrument, acquiring a second language, playing video games, or even staring at lines on a screen - all of these activities have been shown to mold the brain. Meditation is far from unique in this regard, so it makes little sense to appeal to the idea that mindfulness practice changes your brain as a way of proving that it really has effects. If a practice changes subjective experience, it almost certainly changes the brain.

What remains less obvious, however, is whether current brain imaging methods can accurately pick up and make sense of the changes in the brain brought about by practices such as mindfulness meditation. The scientific evidence that meditative practices leave lasting positive imprints on the brain remains tentative ${ }^{2}$. For one thing, much of the available evidence relies on correlation, not causation. The majority of studies investigating meditation-induced neuroplasticity compare long-term meditators to novices. From these studies alone, we cannot ascertain whether the observed brain differences are really due to the practice of meditation or simply reflect a pre-existing difference between the groups. Perhaps people with thicker brains in certain regions are just more likely to take up the practice of meditation and stick to it.

Neuroscientists who study meditation are well aware of the limitations of merely comparing people who have experience with meditation to those who don't. A handful of studies have tackled this issue head on by employing controlled longitudinal designs. In these studies, researchers track participants over time as they learn to meditate. The findings suggest that meditating for just a few weeks, or even days, changes the structure and activity of the brain ${ }^{7}$. Most of these longitudinal studies compare meditation training to a control condition involving a waitlist or practice of a tangential skill such as reading or progressive relaxation. Such controlled designs strengthen the claim that the observed brain changes came about specifically from meditation training. Furthermore, some of the regions that have been shown to change following 
meditation training overlap with the changes observed in meditation experts, lending further support to the idea that these brain areas may play a role in meditation. Stimulating some of these brain areas in mice has even been shown to make these mice exhibit less apparently anxious behavior-leading to the first so-called "rodent model" of meditation (though the model would be better described as a meditation model of rodent behavior $)^{8}$.

Nonetheless, these findings should be taken with a grain of salt. Crucially, most of the neuroimaging studies on meditation have very small sample sizes. Neuroimaging studies with fewer than 20 participants per group - which includes the vast majority of studies on meditation - have very low statistical power to accurately detect all but the largest of effects in a limited set of brain regions ${ }^{9}$. Low statistical power not only increases the chances of missing real effects (type II error), but also substantially inflates the risk of finding spurious results that nonetheless pass statistical threshold (type I error)—see chapter 19 for more detail on this topic. Findings from small samples should really be treated as preliminary until they are replicated in independent groups with more statistical power. Yet, few of the longitudinal findings on brain changes in meditation have been replicated. Compound this situation with the trendiness of meditation science and the fact that negative findings often go unpublished, and you have the perfect recipe for a field at high risk of false positives ${ }^{10}$.

One way to partially mitigate these concerns is through meta-analysis, an approach that amalgamates all of the available data in a scientific field to see if reliable patterns emerge. A few recent meta-analyses have suggested that there may be some consistent brain changes across neuroimaging studies of meditation ${ }^{6,11}$. However, the software that was used to conduct these meta-analyses had a bug that accidentally inflated statistical effects ${ }^{12}$. Unfortunately, the metaanalyses were published before this bug was discovered and fixed, so there is good reason to think that the results may be overstated. This is a poignant illustration of the complex statistical and interpretive issues that permeate a young scientific field such as neuroimaging ${ }^{13}$. Given the tenuous nature of the neuroscientific evidence concerning meditation, it seems misguided to think that the reported brain changes are more meaningful than other observable effects, such as changes in behavior, subjective experience, or clinical symptoms.

\section{The mindful brain in social and bodily context}

Even if we assume that the brain changes reported in neuroimaging studies of meditation are robust, there remains a deeper conceptual problem with the idea that we can map (let alone reduce) complex behaviors or mental processes to changes in particular regions or networks of the brain ${ }^{14}$. There is more to mindfulness than just adopting a brain state or training a brain pattern. Mindfulness is not an internal cognitive process that maps neatly onto the brain; it's a complex orchestration of cognitive skills embodied in a particular social context ${ }^{5,15}$. 
Consider parenting as an analogy. Parenting skills certainly depend on the brain — and practicing them changes the brain - but they are not private mental processes and do not exist inside the brain. Specific patterns of brain activity might correlate with being a good parent in a given context, but these brain patterns alone hardly explain what it is to be a good parent. Good parenting isn't inside the brain; it's a way in which the whole person (including the brain) is engaged in the world. Moreover, what counts as good parenting differs depending on the culture. So appealing to the brain simply won't tell us what it means to be a good parent. To bring this into view we need a wider perspective, one that takes into consideration the context of the whole person as well as the social and cultural environment. The same is true for mindfulness.

Social context shapes mindfulness meditation in many ways: it influences what people hope to achieve from meditating, how they arrange their environment and body when they practice, and who else is around when they meditate - to name but a few examples. All of these social factors likely impact meditation experience in a multitude of ways that scientists are only just beginning to understand ${ }^{15,16}$. For example, one recent study examined how expectations influence the outcomes of a simple mindful breath awareness exercise ${ }^{17}$. Before the mindfulness exercise, the researchers told half of the participants (all of whom were new to meditation) that the practice would immediately improve their attention skills. The other participants were told instead that the meditation would exhaust the brain's limited attention capacity. Only participants who were led to expect positive changes showed improvements in attention (in fact, negative expectations actually impaired attention performance). This finding highlights the importance of social beliefs and expectations in shaping the outcomes of mindfulness practice ${ }^{18}$. It's not just the instructions we follow that determine our experience of meditation. It's also the expectations and beliefs we glean from our social context. The "meaning" we ascribe to practices, including mindfulness meditation, seems to play a key role in shaping our thinking, our healing, and even our biology ${ }^{19}$.

The body also plays a crucial role in mindfulness meditation ${ }^{20,21}$. Many traditions of meditative practice consider the posture of the body to act as a mirror of the mind ${ }^{22}$. When attention dulls, the posture slackens. When thinking becomes agitated or aggressive, the muscles become stiff and taut. Mind and body are bound together. Modern neuroscientific studies support this key insight of ancient meditative traditions. Recent findings demonstrate that bodily posture (e.g., sitting upright versus lying down) profoundly alters baseline brain activity ${ }^{23}$. Moreover, some of the brain regions that are most susceptible to posture-dependent changes are the same regions that are often linked to meditation (such as regions of the "default-mode network"). The very act of lying down for an fMRI scan, or sitting down to meditate, changes how the brain operates (see chapter 12). How then can we hope to understand the meditating brain without appreciating the meditating body?

\section{Conclusion}


The popularity of the mindful brain says a great deal about our culture's fascination with scientific evidence and our eagerness to reduce the richness of subjective experience to a physical substrate that we can see and measure. The allure of neuroimaging feeds into this neurocentric view of mindfulness, which in turn reinforces our internally-focused approach to well-being. But mental wellness depends on more than what's inside our heads. Moving past a neurocentric view of mindfulness promises not only to improve the science of meditation, but also to counteract the pernicious idea that taking care of our minds is just a matter of regulating our own internal states. Part of what meditative practice reveals is that our minds are intrinsically tied up with our bodies and with the larger social and ecological contexts in which we are embedded. We hope for a science of mindfulness that would make us more, not less, mindful of how our brains fit into this bigger picture.

\section{Additional readings:}

An in-depth exploration of the central ideas in this chapter:

Thompson E. Meditation, Buddhism, and science. New York: Oxford University Press: 2017.

Chapter 3, Looping effects and the cognitive science of mindfulness meditation; p. 47-61.

A historian addressing the importance of cultural context as a mechanism in meditation: McMahan DL. Meditation, Buddhism, and science. New York: Oxford University Press: 2017. Chapter 2, How meditation works: Theorizing the role of cultural context in Buddhist contemplative practices; p. 21-46.

A journal issue dedicated to thinking about how cultural context shapes the experiences and outcomes of meditative practices:

Kirmayer LJ. Mindfulness in cultural context. Transcult Psychiatry, 2015;52(4):447-469.

A scientific review arguing for the importance of the body in meditation:

Khoury B, Knäuper B, Pagnini F, Trent N, Chiesa A, Carrière K. Embodied mindfulness. Mindfulness. 2017 Oct;8(5):1160-71.

\section{References}

1. Purser RE, Forbes D, Burke A, editors. Handbook of Mindfulness: Culture, Context, and Social Engagement. Springer International Publishing; 2016.

2. Van Dam NT, van Vugt MK, Vago DR, Schmalzl L, Saron CD, Olendzki A, Meissner T, Lazar SW, Kerr CE, Gorchov J, Fox KC. Mind the hype: A critical evaluation and prescriptive agenda for research on mindfulness and meditation. Perspect Psychol Sci. 2018 Jan;13(1):36-61. 
3. Congleton C, Hölzel BK, Lazar SW. Mindfulness can literally change your brain. Harvard Bus Rev. 2015 Jan 8:309-18.

4. Begley S. Train your mind, change your brain: How a new science reveals our extraordinary potential to transform ourselves. Random House; 2007.

5. Thompson E. Meditation, Buddhism, and science. New York: Oxford University Press: 2017. Chapter 3, Looping effects and the cognitive science of mindfulness meditation; p. 47-61.

6. Fox KC, Nijeboer S, Dixon ML, Floman JL, Ellamil M, Rumak SP, Sedlmeier P, Christoff K. Is meditation associated with altered brain structure? A systematic review and meta-analysis of morphometric neuroimaging in meditation practitioners. Neurosci Biobehav R. 2014 Jun $1 ; 43: 48-73$.

7. Tang YY, Hölzel BK, Posner MI. The neuroscience of mindfulness meditation. Nat Rev Neurosci. 2015 Apr;16(4):213.

8. Weible AP, Piscopo DM, Rothbart MK, Posner MI, Niell CM. Rhythmic brain stimulation reduces anxiety-related behavior in a mouse model based on meditation training. P Natl Acad Sci USA. 2017 Mar 7;114(10):2532-7.

9. Button KS, Ioannidis JP, Mokrysz C, Nosek BA, Flint J, Robinson ES, Munafò MR. Power failure: why small sample size undermines the reliability of neuroscience. Nat Rev Neurosci. 2013 May; 14(5):365.

10. Munafò MR, Nosek BA, Bishop DV, Button KS, Chambers CD, du Sert NP, Simonsohn U, Wagenmakers EJ, Ware JJ, Ioannidis JP. A manifesto for reproducible science. Nat Hum Behav. 2017 Jan 10;1:0021.

11. Fox KC, Dixon ML, Nijeboer S, Girn M, Floman JL, Lifshitz M, Ellamil M, Sedlmeier P, Christoff K. Functional neuroanatomy of meditation: A review and meta-analysis of 78 functional neuroimaging investigations. Neurosci Biobehav R. 2016 Jun 30;65:208-28.

12. Eickhoff SB, Laird AR, Fox PM, Lancaster JL, Fox PT. Implementation errors in the GingerALE Software: description and recommendations. Hum Brain Mapp. 2017 Jan 1;38(1):711.

13. Poldrack RA, Baker CI, Durnez J, Gorgolewski KJ, Matthews PM, Munafò MR, Nichols TE, Poline JB, Vul E, Yarkoni T. Scanning the horizon: towards transparent and reproducible neuroimaging research. Nat Rev Neurosci. 2017 Feb;18(2):115-126.

14. Choudhury S, Slaby J. Critical neuroscience: A handbook of the social and cultural contexts of neuroscience. John Wiley \& Sons; 2012. 
15. McMahan DL. Meditation, Buddhism, and science. New York: Oxford University Press: 2017. Chapter 2, How meditation works: Theorizing the role of cultural context in Buddhist contemplative practices; p. 21-46.

16. Kirmayer LJ. Mindfulness in cultural context. Transcult Psychiatry, 2015;52(4):447-469.

17. Prätzlich M, Kossowsky J, Gaab J, Krummenacher P. Impact of short-term meditation and expectation on executive brain functions. Behav Brain Res. 2016 Jan 15;297:268-76.

18. Farb NA. Mind your expectations: exploring the roles of suggestion and intention in mindfulness training. Journal of Mind-Body Regulation. 2012 Jul;2(1):27-42.

19. Moerman DE, Jonas WB. Deconstructing the placebo effect and finding the meaning response. Ann Intern Med. 2002 Mar;136(6):471-6.

20. Khoury B. Mindfulness: Embodied and embedded. Mindfulness. 2017 Nov:1-6.

21. Khoury B, Knäuper B, Pagnini F, Trent N, Chiesa A, Carrière K. Embodied mindfulness. Mindfulness. 2017 Oct;8(5):1160-71.

22. Johnson W. The posture of meditation: A practical manual for meditators of all traditions. Shambhala Publications; 1996.

23. Lifshitz M, Thibault RT, Roth RR, Raz A. Source localization of brain states associated with canonical neuroimaging postures. J Cognitive Neurosci. 2017 Jul;29(7):1292-301. 